\title{
Analysis of spatial pattern of Frankliniella occidentalis (Thysanoptera: Thripidae) on greenhouse cucumbers using dispersion index and spatial autocorrelation
}

\author{
Kijong Cho, * Joon-Ho Lee, ${ }^{1}$ Jung-Joon Park, Jong-Kwan Kim and Ki-Baik Uhm² \\ Department of Agricultural Biology, Korea University, 1, 5-ka, Anam-dong, Sungbuk-ku, Seoul, 136-701, Korea \\ ${ }^{1}$ Department of Agricultural Biology, School of Agricultural Biotechnology, Seoul National University, Suwon, 441-744, Korea \\ ${ }^{2}$ Division of Entomology, National Institute of Agricultural Science and Technology, Rural Development Administration, Suwon, \\ 441-707, Korea
}

(Received 21 March 2000; Accepted 18 September 2000)

\begin{abstract}
Studies were conducted in two commercial cucumber greenhouses to examine thrips' seasonal and spatial patterns on Cheju Island, Korea, in 1996. Leaf and flower samples were taken to determine the thrips species and stage complexes inhabiting cucumber plants. Adult thrips (54-55\%) were the most dominant stage on flowers and the majority of adult species was Frankliniella occidentalis (Pergande), whereas immature (82-84\%) was the dominant stage on leaves. To determine the spatial distribution patterns of thrips, at least 63 leaf samples which were regularly spaced within a greenhouse were visually inspected from among the 1st, the 7th and the 15th leaves assigned from the top plant canopy. The leaf positions were located at 1.8, 1.0 and $0.3 \mathrm{~m}$ above ground level, respectively. Taylor's power law indicated that the counts of thrips on leaves were aggregated, regardless of thrips stage and leaf position. Autocorrelation analysis, which is based on the relative position of samples revealed the different spatial distribution patterns among the leaf positions. In general, the counts of thrips were nonrandomly distributed on the 7th leaf position, whereas the counts were randomly distributed on the 1st and 15th leaf positions. Correlograms suggested the presence of single or multiple gradients within the sample portion of the greenhouse, depending on thrips stage. Our results suggest that Taylor's power law cannot detect the spatial relationship in data sets accurately, and testing correlograms for significance is more accurate for describing the spatial distribution patterns of thrips.
\end{abstract}

Key words: Frankliniella occidentalis, cucumber, Taylor's power law, autocorrelation, Moran's I

\section{INTRODUCTION}

All organisms are discrete entities that mainly interact with neighboring individuals of their own or other species (Tilman et al., 1997). The importance of spatial heterogeneity comes from its central role in ecological theories and its practical role in population sampling theory and in the development of rational pest management strategies (Legendre and Fortin, 1989). For these reasons, a great deal of effort has been invested in characterizing spatial patterns of insect densities (Liebhold et al., 1991). Traditionally, patterns of spatial dispersion in insects have been described using dispersion indices such as Taylor's power law (Taylor, 1961) and Iwao patchiness regression (Iwao, 1968). However, these indices focus on among sample count variance and ignore the spatial location of the samples
(Liebhold et al., 1991). This property produces certain undesirable effects: the indices sometimes fail to differentiate among different spatial patterns (Midgarden et al., 1993).

Unlike dispersion indices, geostatistical methods use the information contained in the location of individual values in a data set to describe and model the spatial relationship of these values. The spatial autocorrelation is a geostatistical method widely used to explain biological phenomena. The autocorrelation coefficient for each set of data values whose locations are separated by a common distance are plotted against that distance and the spatial relationship of data can be observed (Legendre, 1993).

Recently introduced thrips species, Frankliniella occidentalis (Pergande) and Thrips palmi Karny, have become serious pests in greenhouse cucum-

\footnotetext{
* To whom correspondence should be addressed.
} 
bers in Korea (Cho et al., 1998). These thrips species can cause direct damage to cucumber flowers and fruits and the resulting fruits are malformed or scarred (Rosenheim et al., 1990; Higgins, 1992). Direct larval feeding of leaves can also profoundly reduce cucumber production (Steiner, 1990). A primary control strategy for thrips is the application of insecticides and the use of crop sanitation as a preventive measure because no effective biological control agents are currently available in Korea. However, these thrips species are difficult pests to manage because few effective and registered pesticides are available for control in Korea (Cho et al., 1999).

An understanding of the spatial distribution patterns and crop colonizing trends of phytophagous thrips is expected to provide important information that could be used in the design of sampling schemes and an integrated pest management (IPM) system for thrips in cucumber greenhouses. The aim of the current study is to determine the species composition and seasonal abundance of thrips colonizing cucumbers in Korea, and to analyze the spatial relationship of $F$. occidentalis on cucumber plants by dispersion index and geostatistical methods.

\section{MATERIALS AND METHODS}

Thrips populations were monitored in two commercial cucumber greenhouses (GA and GB) located near Cheju City $\left(33^{\circ} 30^{\prime}, 126^{\circ} 30^{\prime}\right)$ on Cheju Island, Korea, from May to July of 1996. Threeweek-old greenhouse-grown 'Mannungchungjung' cucumber plants were transplanted on 5 March in each greenhouse. Greenhouses were separated from each other by a distance of $11 \mathrm{~km}$. Both greenhouses measured 24 by $45 \mathrm{~m}$ and were surrounded by potato fields.

All greenhouses were managed with standard recommended production practices, including the use of fertilizers and pesticides. Pesticides were used to suppress and prevent insect pests and plant diseases, at the grower's discretion. Surveyed greenhouses consisted of $12-15$ beds on $\approx 0.8 \mathrm{~m}$ centers and plants were grown using the modified vertical cordon training system (Cho et al., 1998). Horizontal support wires were positioned directly over the row of plants, at a height of $1.8-2.0 \mathrm{~m}$. Initially each plant was trained vertically along and around the support plastic twine and fastened with plastic snap-on clips. As the plant reached the top supporting wire, the grower removed the clips and released the reserved twine, leaving the plant $\approx 0.3 \mathrm{~m}$ closer to the ground, with its lower section lying on the ground. Therefore, the youngest leaf always occurred at the top canopy.

Plant samples. Plant parts were sampled to obtain the thrips species compositions that occurred on the cucumbers. Cucumber flower and leaf samples were taken weekly or biweekly from the middle stratum (1.2 $\mathrm{m}$ above ground level) of the plant using the same methods described by Higgins (1992). The sample size for each plant part should be limited, because sampling is destructive (potential fruits and carbon assimilation sources are removed). For flower samples, 3-5 vials, each containing two flowers, were collected for each sampling date and greenhouse. For leaf samples, 3-4 samples were collected and processed using a washing method (Higgins, 1992).

Adult thrips species from the flower and leaf samples were identified to species level with the aid of a dissecting microscope. Because no keys were available for identifying immature thrips to the species level, all immatures were combined into a single group. Statistical analysis of thrips abundance in each plant part was not performed because sample size varied among the samples. The results reported here show the total numbers of adult thrips species and immatures for each greenhouse.

Visual estimates. Cucumber leaves were visually inspected weekly to obtain the seasonal and spatial changes in thrips population density. For seasonal abundance of thrips, at least 30 plants were selected in a random manner and the numbers of adult and immature thrips from the1st, the 7th and the 15th leaves from the upper canopy level were counted. Each leaf position was located $\approx 1.8$, $\approx 1.0$ and $\approx 0.3 \mathrm{~m}$ above ground level, respectively. The 1st was a newly expanded leaf behind the growing tip, and the 7th and 15th were fully matured non-senesced leaves. The choice of leaf categories reflects both the leaf age and vertical canopy height. The 1 st and the 15th positions represented the youngest and the oldest leaves, respectively.

For analysis of the spatial distribution patterns of thrips, an approximate quadrat of permanent sampling stations was established for each greenhouse. 
The sampling array for each greenhouse consisted of 72 visual estimates, laid out in a $8 \times 9$ quadrat pattern. Each quadrat covered $\approx 15 \mathrm{~m}^{2}$ and contained $\approx 50$ plants. One cucumber plant located at the center of the quadrat was selected and the numbers of adult and immature thrips were counted for each leaf position and sampling date. Distance between the plant stations was $\approx 3 \mathrm{~m}$ in a down-row and $\approx 5 \mathrm{~m}$ in a cross-row. Houses GA and GB were surveyed 7 and 6 times, respectively, throughout the growing season for the analysis of spatial patterns of thrips.

Identification of the thrips to species level was not attempted because it is not feasible to identify thrips to the species level in the field without the aid of a dissecting microscope.

\section{Spatial analysis}

Dispersion index. Taylor (1961) showed that variance is related to mean density such that $\log s^{2}$ $=\log a+b \log \bar{x}$, where the slope $(b)$ is a measure of aggregation. The mean densities $(\bar{x})$ of adult and immature thrips per leaf position and the variances $\left(s^{2}\right)$ were calculated for each greenhouse. The general linear regression model procedure (PROC GLM) of SAS (SAS Institute, 1992) was used to estimate the regression parameters. Homogeneity of equality of slopes from the Taylor regression for counts of adult and immature thrips among different plant positions was tested with an analysis of covariance (ANCOVA) (Sokal and Rohlf, 1995).

Geostatistical analysis. The Moran's I index, which is a common measure of spatial autocorrelation statistic (Cliff and Ord, 1981), is used for spatial analysis. This index, scaled between +1 and -1 , incorporates the similarity among values with the similarity among location or distance (Midgarden et al., 1993). Moran's $\boldsymbol{I}$ is calculated from values separated by a given lag distance $(h)$ using the formula:

$$
\begin{aligned}
\text { Moran's } \boldsymbol{I}_{h}= & \left(n / \sum C_{i j}\right)\left[\sum \sum C_{i j}\left(z_{i}-z_{\text {mean }}\right)\right. \\
& \left.\times\left(z_{i}-z_{\text {mean }}\right) / \sum\left(z_{i}-z_{\text {mean }}\right)^{2}\right],
\end{aligned}
$$

where $n$ is the number of samples; $C_{i j}=1$ if the two samples are separated by lag distance $h$ and $C_{i j}=0$ if they are not separated. The $z_{i}$ and $z_{j}$ are values of a variable at location $i$ and location $j$, respectively. In this study, observations refer to the numbers of adult or immature thrips at a given sampling station and date.
A positive Moran's I indicates that values separated by a given lag distance tend to be similar (positive spatial autocorrelation), whereas a negative Moran's $I$ indicates that values tend to be dissimilar (negative spatial autocorrelation) when separated by that distance. The expected value of $\boldsymbol{I}$ in the absence of significant spatial autocorrelation is around 0 (Cliff and Ord, 1981). For a correlogram, plots of autocorrelation coefficients as a function of distance classes, to be globally significant, at least one value of $\boldsymbol{I}$ has to be significant at an alpha level of $0.05 / k$, where $k$ represents the number of distance classes considered, according to Bonferroni's method of correcting for multiple tests (Legendre and Fortin, 1989). Once this is established, individual values of $\boldsymbol{I}$ can be declared significant at the 0.05 level.

Spatial autocorrelation statistics were estimated using the software of SAAP (spatial autocorrelation analysis program) (Wartenberg, 1989). Seven equal distance classes of $6.5 \mathrm{~m}$ with mutually exclusive intervals were established. The boundaries of these intervals were chosen in such a way that the largest distance class will include a minimum of 100 pair points to allow the calculation of $\boldsymbol{I}$ for each successive distance class from a sufficient number of point pairs.

\section{RESULTS}

\section{Thrips species compositions on cucumber flow- ers and leaves}

There were profoundly different thrips stage compositions between the plant parts (Table 1). A higher proportion of adults to total number of thrips was collected from the flower than from the leaf samples in both greenhouses. The range of adult proportions was $54.3-55.3 \%$ on flowers, whereas it was $15.4-17.8 \%$ on leaves. The opposite was true for immature thrips.

$F$. occidentalis was the most abundant adult thrips species, regardless of greenhouse and plant part sampled (Table 2). This species consisted of at least $83.3 \%$ of the total adult thrips collected from flowers and leaves. Most of the remaining adults were Thrips tabaci Lindeman, which were only collected from cucumber leaves. T. palmi, Frankliniella intonsa (Trybon) and Thrips nigropilosis Uzel accounted for $<2.2 \%$ of the total thrips collected (Table 2). 
Table 1. Total number of adult and immature thrips collected from cucumber leaves and cucumber flowers from two commercial greenhouses (GA and GB) on Cheju Island, Korea, in 1996

\begin{tabular}{|c|c|c|c|c|}
\hline \multirow{2}{*}{ Stage } & \multicolumn{2}{|c|}{ GA } & \multicolumn{2}{|c|}{ GB } \\
\hline & Leaf & Flower & Leaf & Flower \\
\hline Adult & $90(17.8)^{\mathrm{a}}$ & $478(55.3)$ & $398(15.4)$ & $2,133(54.3)$ \\
\hline Immature & $417(82.2)$ & $386(44.7)$ & 2,191 (84.6) & $1,796(45.7)$ \\
\hline Total & 507 & 864 & 2,589 & 3,929 \\
\hline
\end{tabular}

${ }^{a}$ Values in parentheses are the percentage of thrips stage collected from cucumber leaves and flowers.

Table 2. Total number of adult thrips species collected from cucumber leaves and cucumber flowers from two commercial greenhouses (GA and GB) on Cheju Island, Korea, in 1996

\begin{tabular}{|c|c|c|c|c|}
\hline \multirow{2}{*}{ Species } & \multicolumn{2}{|c|}{ GA } & \multicolumn{2}{|c|}{ GB } \\
\hline & Leaf & Flower & Leaf & Flower \\
\hline F. occidentalis & $75(83.3)^{\mathrm{a}}$ & $464(97.1)$ & $384(96.5)$ & $2,111(99.0)$ \\
\hline F. intonsa & $1(1.1)$ & $14(2.9)$ & $0(0.0)$ & $4(0.2)$ \\
\hline T. tabaci & $7(7.8)$ & $0(0.0)$ & $11(2.8)$ & $0(0.0)$ \\
\hline T.palmi & $2(2.2)$ & $0(0.0)$ & $0(0.0)$ & $0(0.0)$ \\
\hline Others ${ }^{\mathrm{b}}$ & $5(5.6)$ & $0(0.0)$ & $3(0.7)$ & $18(0.8)$ \\
\hline
\end{tabular}

${ }^{a}$ Values in parentheses are the percentage of adult thrips species collected from cucumber leaves and flowers.

${ }^{\mathrm{b}}$ Other thrips included Frankliniella intonsa (Trybon), Thrips nigropilosis Uzel and unidentified thrips.

\section{Visual estimate of thrips}

Significantly higher numbers of adult and immature thrips were observed from the 7th leaf position compared to the 1st and 15th leaf positions in all greenhouses $(p<0.05)$. The seasonal trends from the 7th leaf position are only presented (Fig. 1) because the changes of thrips populations were comparable among the leaf positions in each greenhouse. Immatures outnumbered adult thrips throughout the growing season and were responsible for most of the population changes on cucumber plants for both greenhouses. The numbers of adult thrips remained relatively constant with low densities.

\section{Spatial analysis}

Dispersion index. All $b$ values of Taylor's power law were $>1$, indicating aggregated distribution regardless of thrips stage, leaf position and greenhouse surveyed (Table 3). A comparison of $b$ values for adult and immature thrips from each leaf position showed that these values did not differ significantly between the thrips stages $(p>0.05)$. ANCOVA revealed that none of the $b$ values from dif-

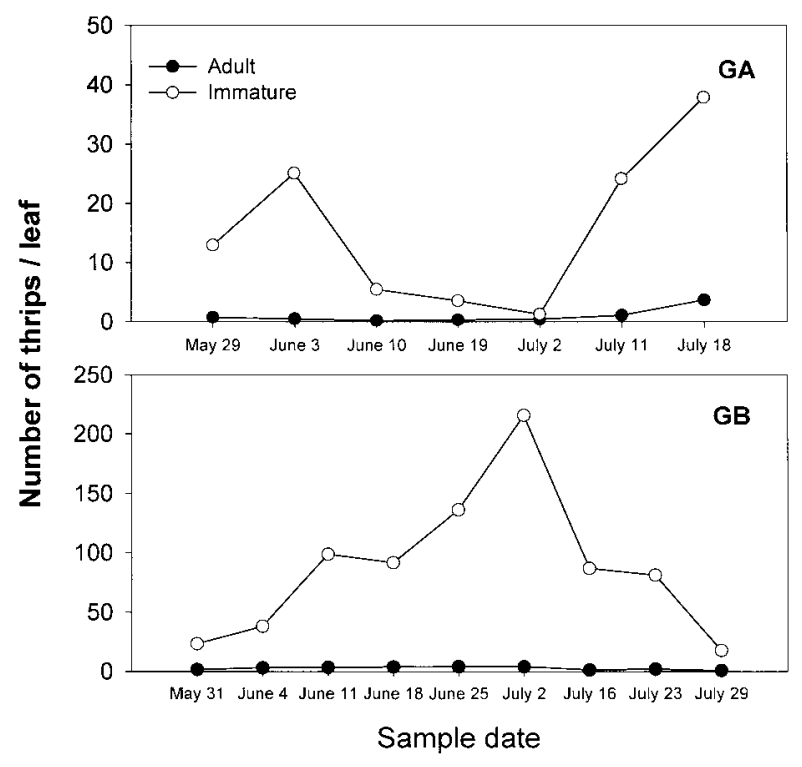

Fig. 1. Visual mean estimates of adult and immature thrips on the 7th leaf position of cucumber plants from two cucumber greenhouses (GA and GB) in 1996.

ferent leaf positions differed significantly at either of the greenhouses $(p>0.10)$, indicating that each thrips stage had a similar mean-to-variance rela- 
Table 3. Regression statistics of Taylor's power law relationship for adult (A) and immature (I) thrips sample data collected from three different leaf positions in cucumber greenhouses (GA and GB) during 1996, Cheju Island, Korea

\begin{tabular}{ccccccc}
\hline Greenhouse & Leaf position $^{\mathrm{a}}$ & Stage & $n^{\mathrm{b}}$ & $b^{\mathrm{c}} \pm$ SEM & $r^{2}$ & Mean density ranges \\
\hline \multirow{2}{*}{$\mathrm{GA}$} & \multirow{2}{*}{1 st } & A & 7 & $1.56 \pm 0.19$ & 0.94 & $0.01-1.29$ \\
& & I & 7 & $1.67 \pm 0.18$ & 0.94 & $0.52-6.55$ \\
& & A & 7 & $1.64 \pm 0.17$ & 0.94 & $0.22-3.72$ \\
& \multirow{2}{*}{15 th } & I & 7 & $1.63 \pm 0.18$ & 0.94 & $1.29-37.86$ \\
& & A & 7 & $1.56 \pm 0.08$ & 0.98 & $0.11-3.50$ \\
& I & 7 & $1.72 \pm 0.05$ & 0.99 & $0.42-20.86$ \\
\hline \multirow{2}{*}{ GB } & A & 6 & $1.70 \pm 0.71$ & 0.65 & $0.38-1.22$ \\
& \multirow{2}{*}{7 th } & I & 6 & $1.62 \pm 0.24$ & 0.86 & $0.46-14.05$ \\
& A & 6 & $1.44 \pm 0.22$ & 0.75 & $0.80-4.16$ \\
& 15th & I & 6 & $1.67 \pm 0.10$ & 0.97 & $17.76-215.91$ \\
& & I & 6 & $1.70 \pm 0.33$ & 0.77 & $0.52-6.94$ \\
& & 6 & $1.50 \pm 0.29$ & 0.71 & $17.63-165.80$ \\
\hline
\end{tabular}

${ }^{\mathrm{a}}$ Leaf positions were assigned from top canopy of cucumber plant.

${ }^{\mathrm{b}}$ The numbers of $\bar{x}$ and $s^{2}$ pairs used to calculate regression statistics.

${ }^{\mathrm{c}}$ All $b$ values are significantly $>1(p<0.05)$ according to a $t$-test.

Table 4. Number of sample-weeks with a globally significant spatial relation as determined by analyzing spatial autocorrelation for adult (A) and immature (I) thrips in cucumber greenhouses (GA and GB)

\begin{tabular}{|c|c|c|c|c|}
\hline Leaf position ${ }^{\mathrm{a}}$ & Greenhouse & Stage & No. of sample-weeks & $\begin{array}{l}\text { No. of sample-weeks with } \\
\text { significant spatial relations }{ }^{\mathrm{b}}(\%)\end{array}$ \\
\hline \multirow[t]{4}{*}{1 st } & GA & A & 7 & $1(14)$ \\
\hline & & I & 7 & $1(14)$ \\
\hline & GB & A & 6 & $0(0)$ \\
\hline & & I & 6 & $3(50)$ \\
\hline \multirow[t]{4}{*}{ 7th } & GA & A & 7 & $5(71)$ \\
\hline & & I & 7 & $5(71)$ \\
\hline & GB & A & 6 & $4(67)$ \\
\hline & & I & 6 & $6(100)$ \\
\hline \multirow[t]{4}{*}{ 15th } & GA & A & 7 & $1(14)$ \\
\hline & & I & 7 & $1(14)$ \\
\hline & GB & A & 6 & $1(17)$ \\
\hline & & I & 6 & $2(33)$ \\
\hline
\end{tabular}

${ }^{a}$ Leaf positions were assigned from top canopy of cucumber plant.

${ }^{\mathrm{b}}$ Correlograms as a whole were tested for deviation from a null hypothesis of no spatial relation using Bonferroni's approximation (Legendre and Fortin, 1989).

tionship within cucumber plants. Further ANCOVA comparing the $b$ values from two greenhouses showed no significant differences $(p>0.10)$.

Geostatistical analysis. Spatial autocorrelation analysis showed that few spatial relations (Bonferroni's approximation at alpha level of 0.05) of adults and immatures were found from the 1 st and the 15th leaf positions (Table 4), despite the fact that their $b$ values from Taylor's power law implied an aggregated spatial distribution (Table 3). However, higher spatial relations for adults and immatures were observed from the 7th leaf position compared to other positions with the exception of immature populations on the 15 th leaf position 


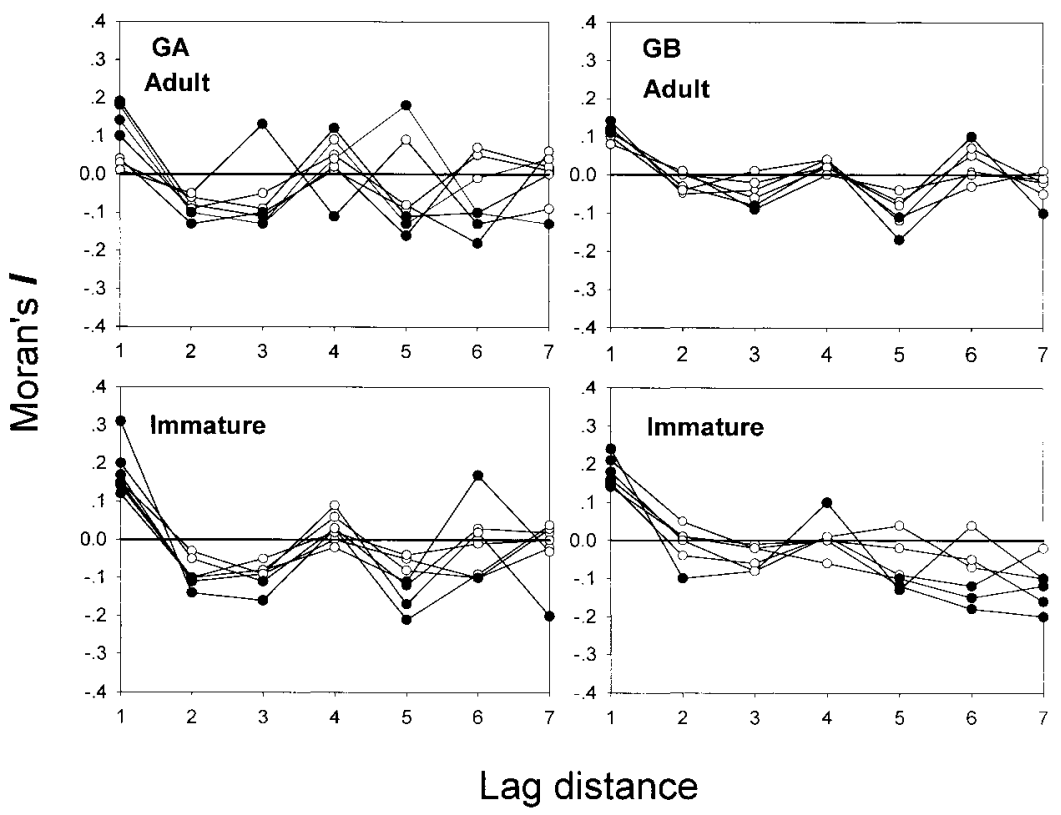

Fig. 2. Spatial correlograms of adult and immature thrips on the 7th leaf position of cucumber plants from two cucumber greenhouses (GA and GB) in 1996. Significant values $(p<0.05)$ of Moran's $I$ are indicated by black circles; open circles represent nonsignificant values.

from house GB.

Only correlograms from the 7th leaf position in each greenhouse were illustrated to compare the spatial distribution patterns between thrips stages (Fig. 2), because most spatial relations of thrips count data were found at this leaf position (Table 4). In nearly all cases, Moran's I was positive and significant at the first lag distance for all thrips stages in both greenhouses. Moran's I values between the second and the third lag distances were generally negatively autocorrelated and returned to positive values at the fourth lag distance. A similar trend was observed beyond the fourth lag distance except for immature populations in house GB.

\section{DISCUSSION}

$F$. occidentalis, one of the most problematic pests in greenhouses, had the most thrips species on cucumber flowers and leaves for both greenhouses (Table 2). F. occidentalis is of particular concern in cucumber greenhouses because this species is the vector of tomato spotted wilt virus (Lewis, 1973) and is insecticide resistant (Immaraju et al., 1992). Adult F. occidentalis primarily inhabit cucumber flowers and are responsible for the majority of cucumber fruit damage (Childers, 1997). Most immatures found in flowers probably moved from nearby leaves to gain access to cucumber floral nectar because most of their eggs were laid on leaves (Higgins, 1992). This explains why a relatively low proportion (44.7$45.7 \%$ ) of immatures was found on flowers, compared to the proportion $(82.2-84.6 \%)$ on leaves (Table 1).

The immature populations from the 7th leaf mostly determined population changes of thrips in all cucumber greenhouses (Fig. 1). It was also true for adult thrips that higher numbers were found on the matured cucumber leaves (e.g., the 7th and the 15th leaves). Steiner (1990) reported similar results that adults and immatures of $F$. occidentalis favored the older cucumber leaves over the younger leaves. However, Kawai (1990) reported that adults of $T$. palmi on cucumber plants are usually found on the upper canopy (younger cucumber leaves), while immatures are on the lower canopy (older cucumber leaves). These leaf preference differences between thrips species and stage cannot be explained in this study and additional studies are necessary to elucidate these phenomena.

Values for aggregation coefficient $b$ of Taylor's power law suggest that the distribution patterns of the counts of thrips on cucumber plants are aggregated and consistent among leaf position and thrips stage according to ANCOVA (Table 3). Further- 
more, these $b$ values were not different among the greenhouses surveyed. Steiner (1990) demonstrated a similar finding that $F$. occidentalis was aggregated on cucumber plants, regardless of the leaf age and thrips life stage. These indicate the sample values from the different leaf positions and thrips stages have the same effect on Taylor's power law (Table 3).

The spatial patterns of thrips on cucumber plants are different and were reflected in distinctively different correlograms (Fig. 2 and Table 4). It could be clearly seen that different correlograms were generated from the population, which had similar values of dispersion index (Fig. 2). The majority of globally significant correlograms was detected on thrips count data from the 7 th leaf position and ranged from 67 to $100 \%$ of data sets from houses GA and GB, respectively. This suggests that Taylor's power law does not detect the spatial relation in data sets and testing correlograms for significance is more accurate for describing the spatial distribution patterns of thrips.

The correlograms of houses GA and GB from the 7th leaf position reflect a multiple gradient spatial pattern of thrips with the exception of immatures from house GB, which shows a single gradient pattern (Fig. 2). Values of Moran's I varying from significant positive to negative values suggest a repeated spatial structure in the form of a multiple gradient pattern (Houle, 1998). That correlograms decreased gradually as the lag distance increased indicates a single gradient spatial pattern (Legendre and Fortin, 1989). Regardless of spatial structures, the sample values tended to be similar to each other at the first lag distance (positive spatial autocorrelations) (Fig. 2). Correlograms with significant positive values of $\boldsymbol{I}$ at small distance classes are a common biological phenomenon (Nestel and Klein, 1995; Houle, 1998).

The dispersion pattern of thrips identified in this study poses important issues for the development of an effective thrips IPM program. The importance of spatial heterogeneity comes from its practical role in the population sampling procedure and its central role in understanding the predator-prey relationship (Bierzychudek, 1988; Legendre and Fortin, 1989). Taylor's power law has been utilized for the development of thrips sampling plans on various crops (Steiner, 1990; Cho et al., 1999) using Green's (1970) method. Taylor's power law should be calculated under the assumption that individual sample values are spatially independent (Midgarden et al., 1993) because Taylor's power law did not account for the relative position of sample values within data sets. Therefore, samples should be independent of each other to avoid biased estimates of mean densities in the sampling plans based on the coefficients of Taylor's power law. According to Bonferroni's approximation, at least $67 \%$ of total sample-weeks from the 7 th leaf position had displayed spatial dependency (Table 4). Because the most spatial relations were observed at the first lag, the leaf samples should be $\approx 6.5 \mathrm{~m}$ apart to obtain samples that are less likely to be positively autocorrelated in cucumber greenhouses when the objective is to estimate field means (Fig. 2). Subsequently, according to the resampling simulations (Naranjo and Hutchison, 1997), the nature of spatial relation of the samples affects the performance of the sampling plans in a profound way for adult Trialeurodes vaporariorum (Westwood) on yellow sticky traps in cherry tomato greenhouses (Cho, unpublished data).

Because the highest numbers of thrips were observed on the middle stratum of cucumber plants (Fig. 1), control measures, including biological control, are subject to target the 7th leaf position (i.e., $1.0 \mathrm{~m}$ height from ground level). Numerous arthropods, recognized as predators of phytophagous thrips, have proved their capacity to eliminate or suppress thrips populations in greenhouses and field crops of agricultural importance (Sabelis and Van Rijin, 1997). Many of the experiments have only demonstrated the direct mortality factors of biological control agents against various thrips species. While spatial complexities of thrips and their biological control agents have been kept to a minimum in the biological control programs, they should not be ignored. Spatial analysis shows that the host distribution structures influence the percentage of hosts attacked by parasites, and rates of parasitism were spatially autocorrelated in the forest landscape (Roland and Taylor, 1997). An understanding of spatial distribution patterns of thrips would help to develop a thrips biological control program in greenhouses. A correlogram with significant positive values of Moran's $I$ at small distance classes and nonsignificant values at larger classes suggests patchiness (Houle, 1998). A biological control agent which has a smaller patch size 
compared to thrips species would not suppress thrips populations below the economic threshold level fast enough, if the number of introducing sites for the agent is not large enough to correspond to the patch sizes of thrips. Therefore, the correlogram study can be a good index for determining the numbers of introducing sites of biological control agents in a greenhouse.

\section{ACKNOWLEDGEMENTS}

We thank Sang-Hoon Kang (Cheju RDA, Cheju Do, Korea) for his technical assistance and many hours of field work. Thanks also go to two anonymous reviewers for their critical reviews and English corrections. This study was supported in part by the Korea University Intramural Research Grant for K. Cho.

\section{REFERENCES}

Bierzychudek, P. (1988) Can patchiness promote prey outbreaks? Trends Ecol. Evol. 3: 62-63.

Childers, C. C. (1997) Feeding and oviposition injuries to plants. In Thrips as Crop Pests (T. Lewis ed.). CAB International, Oxon, New York, pp. 505-537.

Cho, K., S. H. Kang and J. O. Lee (1998) Spatial distribution of thrips in greenhouse cucumber and development of a fixed-precision sampling plan for estimating population density. J. Asia-Pacific Entomol. 1: 163-170.

Cho, K., K. B. Uhm and J. O. Lee (1999) Effect of test leaf and temperature on mortality of Frankliniella occidentalis in leaf dip bioassay of insecticides. J. Asia-Pacific Entomol. 2: 69-75.

Cliff, A. D. and J. K. Ord (1981) Spatial Processes: Models and Applications. Pion, London. 266 pp.

Green, R. H. (1970) On fixed precision level sequential sampling. Res. Popul. Ecol. 12: 249-251.

Higgins, C. J. (1992) Western flower thrips (Thysanoptera: Thripidae) in greenhouses: Population dynamics, distribution on plants, and associations with predators. $J$. Econ. Entomol. 85: 1891-1903.

Houle, G. (1998) Seed dispersal and seedling recruitment of Betula alleghaniensis: Spatial inconsistency in time. Ecology 79: 807-818.

Immaraju, J. A., T. D. Paine, J. A. Bethke, K. L. Robb and J. P. Newman (1992) Western flower thrips (Thysanoptera: Thripidae) resistance to insecticides in coastal California greenhouses. J. Econ. Entomol. 85: 9-14.

Iwao, S. (1968) A new regression method for analyzing the aggregation pattern of animal populations. Res. Popul. Ecol. 10: 1-20.

Kawai, A. (1990) Life cycle and population dynamics of Thrips palmi Karny. JARQ 23: 282-288.

Legendre, P. (1993) Spatial autocorrelation: Trouble or new paradigm? Ecology 74: 1659-1673.

Legendre, P. and M.-J. Fortin (1989) Spatial pattern and ecological analysis. Vegetatio 80: 107-138.

Lewis, T. (1973) Thrips: Their Biology, Ecology and Economic Importance. Academic Press, London and New York. 349 pp.

Liebhold, A. M., X. Zhang, M. E. Hohn, J. S. Elkinton, M. Ticehurst, G. L. Benson and R. W. Campbell (1991) Geostatistical analysis of gypsy moth (Lepidoptera: Lymantriidae) egg mass population. Environ. Entomol. 20: 14071411.

Midgarden, D. G., R. R. Youngman and S. J. Fleischer (1993) Spatial analysis of counts of western corn rootworm (Coleoptera: Chrysomelidae) adults on yellow sticky traps in corn: Geostatistics and dispersion indices. Environ. Entomol. 22: 1124-1133.

Naranjo, S. E. and W. D. Hutchison (1997) Validation of arthropod sampling plans using a resampling approach: Software and analysis. Am. Entomol. 43: 48-57.

Nestel, D. and M. Klein (1995) Geostatistical analysis of leafhopper (Homoptera: Cicadellidae) colonization and spread in deciduous orchards. Environ. Entomol. 24: 1032-1039.

Roland, J. and P. D. Taylor (1997) Insect parasitoid species respond to forest structure at different spatial scales. Nature 386: 710-713.

Rosenheim, J. A., S. C. Welter, M. W. Johnson, R. F. L. Mau and L. R. Gusukuma-Minuto (1990) Direct feeding damage on cucumber by mixed-species infestations of Thrips palmi and Frankliniella occidentalis (Thysanoptera: Thripidae). J. Econ. Entomol. 83: 1519-1525.

Sabelis, M. W. and P. C. J. Van Rijin (1997) Predation by insets and mites. In Thrips as Crop Pests (T. Lewis ed.). CAB International, Oxon, New York, pp. 259-354.

SAS Institute (1992) SAS User's Guide. SAS Institute, Cary, NC, pp. 973-975.

Sokal, R. R. and F. J. Rohlf (1995) Biometry. 3rd ed. Freeman, New York. 887 pp.

Steiner, M. Y. (1990) Determining population characteristics and sampling procedures for the western flower thrips (Thysanoptera: Thripidae) and the predatory mite Amblyseius cucumeris (Acari: Phytoseiidae) on greenhouse cucumber. Environ. Entomol. 19: 1605-1613.

Taylor, L. R. (1961) Aggregation, variance and mean. Nature 189: 732-735.

Tilman, D., C. L. Lehman and P. Kareiva (1997) Population dynamics in spatial habitat. In Spatial Ecology (D. Tilman and P. Kareiva eds.). Princeton University Press, Princeton, pp. 3-45.

Wartenberg, D. (1989) SAAP — a spatial autocorrelation analysis program. Department of Environmental and Community Medicine, Robert Wood Johnson Medical School, University of Medicine and Dentistry of New Jersey, Piscataway, NJ. 